I.

\title{
Ein Fall von traumatiseher Exostosis bursata (Abriss der peripheren Sehne des Biceps brachii) nebst Be- merkungen über die Bildung freier Körper in Gelenken und in Bursen.
}

Von

\author{
Dr. Karl Röper.
}

Mit 1 Abbildung auf Tafel I.

Die Unfallgesetzgebung und die Röntgenphotographie haben das wichtige Gebiet der Gelenkchirurgie bedeutend gefördert. Doch sind der Unklarheiten noch genug, insbesondere, was die freien Gelenkkörper angeht. Ihre Diagnose ist meist klar. Die Beschwerden sind sehr charakteristisch, die Palpation gelingt oft, und das Röntgenbild gibt meist genauen Aufschluss. Die Erfolge der Therapie sind dank der Fortschritte der modernen Chirurgie gute zu nennen. Sie besteht unter dem Schutze von A- und Antiseptik in der Eröffnung des Gelenkes und im Herausholen der freien oder gestielt anhaftenden Körper. Unklar ist aber noch nach mancher Seite hin ihre Ätiologie. Ja, diese ist, gerade weil Diagnose und Therapie so vorgeschritten sind, zu einer brennenden Frage geworden, deren augenblicklichen Stand zu erörtern wohl eine reizvolle Aufgabe ist.

Schmaus (1) gibt folgende Definition der freien Gelenkkörper: "Sogenannte freie Gelenkkörper liegen entweder ganz frei im Gelenke oder flottieren, an einem Stiele befestigt, in seiner Höhle." Weil sie oft rasch unter den Fingern des Untersuchenden entschlüpfen, hat sie der Volksmund Gelenkmäuse genannt. Hiervon will Leser (2) von vornherein abgeschieden wissen jene Fibringerinnsel, faserstoffhaltige Massen, welche allmählich sich organisierend zu grösseren Bildungen anwachsen und als Corpora oryzoidea bezeichnet werden. Diese sind als albuminöse Gerinnungen aufzufassen, welche gleich den Sehnenscheidenhygromen, über die König in neuester Zeit mehr Licht verbreitet hat, tuberkulösen Ursprungs sind, d. h. sie finden sich in tuberkulös erkrankten Gelenken teils wandständig, teils von vornherein frei in dem serofibrinösen Ergusse schwimmend. Für diese eine Ausnahmestellung einnehmenden Gelenkkörper ist zuweilen eine kleine Exkreszenz der Synovialwand der erste Anlass zu ihrer Bildung, der erste Kern, um den sich, wie beim Blasensteine die Urate, so hier das Fibrin schichtet. So werden sie wandständig und gestielt. Oft finden sich feine Bindegewebszüge in den übrigens in anderer Hinsicht nicht organisierten Geweben. König sagt mit 
Bezug hierauf: „Die eigentümliche Art von gerinnendem und organisationsfähigem Eiweissexsudat ist in der Tat charakteristisch für Tuberkulose."

Nachdem wir also die Corpora oryzoidea als Fibringerinnungen in tuberkulösen Gelenken abgesondert haben, können wir an die Einteilung der eigentlichen Gelenkkörper, der Corpora libera articulorum gehen. Die ganze Ätiologie dreht sich um die Frage, inwieweit freie Gelenkkörper in vorher gesunden, inwieweit sie in erkrankten Gelenken auftreten. Da hierauf der ganze Streit beruht, so haben auch fast alle Autoren diese Einteilung.

Da können zunächst. Fremdkörper, welche in die Gelenkhöhle gelangt sind, Veranlassung zur Bildung von freien Gelenkkörpern in vorher gesunden Gelenken geben. Meist jedoch ruft der eingedrungene Fremdkörper eine Entzündung hervor, welche die alsbaldige Entfernung des Körpers veranlasst, so dass dieser nicht die Symptome machen kann, die einem Gelenkkörper als solchem eigentümlich sind. Es können aber z. B. abgebrochene Nadelspitzen im Knorpel oder Knochen stecken bleiben und Wucherungsprozesse auslösen. Eine solche Wucherung kann frei werden und als Gelenkmaus imponieren. Nach Fischer (3) war in einem Falle eine Nadelspitze, einmal ein bei einer Operation abgebrochenes Stückchen Säge und einmal ein Schrotkorn das veranlassende Moment.

$\mathrm{Ob}$ ausserdem in vorher gesunden Gelenken freie Gelenkkörper nur durch ein Trauma entstehen können, ist der strittigste Punkt in ihrer Ätiologie. Die letzten Jahrzehnte haben da viele Arbeiten, viele durch Tier- und Leichenexperimente erhärtete Ansichten gebracht, aber für eine Reihe von Fällen muss man sich auch jetzt noch mit einer Hypothese begnügen, und im grossen und ganzen gilt auch heute noch, was Virchow in seiner Geschwulstlehre (4) gesagt hat. Er ist der Ansicht, es müsse genauer geprüft werden, ob von einem gesunden Gelenkende Stücke abbrechen und als Gelenkmäuse auftreten können. Der Autor hält diese Entstehungsweise für unwahrscheinlich, „denn würde z. B. vom Rande eines gesunden Knochens ein Stück abbrechen, so müsste man doch erwarten, dass die ursprüngliche Oberfläche allein überknorpelt wäre, dagegen die Fraktur- oder Fissurstelle entweder bloss kompakten oder spongiösen Knochen oder höchstens eine Um. lagerung durch fibrinöse Niederschläge zeigte. Allein ich fand selbst an Gelenkkörpern der am meisten auffälligen Gestalt fast über die ganze Oberfläche einen Knorpelïberzug, und der scheinbare Knochen erwies sich grossenteils als bloss verkalktes Knorpelgewebe. Dieser Befund stimmt wohl mit der Annahme, dass ursprünglich flache Ekchondrosen von dem Gelenkende des Knochens hervorwuchsen, welche sich den gegenüberliegenden Gelenkenden anpassten und dadurch eine der Artikulation entsprechende Gestalt annahmen, dann teilweise versteinerten und endlich innerhalb des Knorpels abbrachen, aber es lässt sich nicht wohl vereinigen mit der Voraussetzung, dass der Knochen selbst zertrümmert wurde."

Hiermit stimmt Virchow der alten Laennec'schen Lehre zu, dass die freien Gelenkkörper im allgemeinen akzidentelle Knorpel sind, und für die Mehrzahl sei die Ansicht des letzteren richtig, dass sie ursprünglich als ge- 
stielte Auswüchse der Synovialwand anfingen. Die sklerotischen Platten und Auswüchse könnten sich auch vom parietalen Blatte der Synovialis bilden, sie könnten verknorpeln und verknöchern.

Demgegenüber behaupten andere Autoren, freie Gelenkkörper könnten durch Absprengung von Knorpel- und Knochenstücken in einem vorher gesunden Gelenke entstehen. Solche Abrisse sind natürlich bei einer sich ins Gelenk erstreckenden Fraktur möglich, diese aber ist an sich selten. Fast alle Autoren stimmen hierin überein.

Fischer (5) sagt: „Es geht aus der geringen Zahl sicherer Beobachtungen und aus dem ablehnenden Verhalten bewährter Forscher bervor, dass der traumatische Ursprung der Gelenkmäuse in gesunden Gelenken zu den grössten Seltenheiten gehört." $\mathrm{Zu}$ diesen seltenen Fällen einer Absprengungsoder Abschälungsfraktur ist ein von Schild (6) beșchriebener Fall zu rechnen. Es wurde in einem sonst durchaus gesunden Gelenke im Anschlusse an ein Trauma - Fall aufs Ellenbogengelenk - ein freier Gelenkkörper beobachtet. Die bei der Operation (Professor Riedinger) gefundenen Verhältnisse machten es wahrscheinlich, dass es sich um eine Abschälungsfraktur an dem Gelenkende des Humerus handelte. Rinde und Spongiosa des aus richtigem Knochen bestehenden Körpers zeigten die deutlichsten Rückbildungserscheinungen. Von entzündlichen Erscheinungen hatte sich weder an der Oberfläche noch im Inneren des Körpers eine Spur nachweisen lassen.

Helferich (7) sagt über die traumatische Entstehungsweise der freien Gelenkkörper in bezug auf das Kniegelenk: „Wirkt eine Gewalt bei etwas gebeugtem Kniegelenk im Sinne einer Kompression, verbunden mit etwas seitlicher Verschiebung bezw. Drehung, so kann eine Aussprengung eines Knorpelstückchens mit der anhaftenden Spongiosa erfolgen. Die verletzende Gewalt ist dabei öfters auffallend gering, nicht selten nur durch eine ungeschickte Bewegung gegeben. Das Knorpelstück springt scharfrandig heraus, indem der Knorpel in seiner ganzen Dicke steil abbricht. Die Grösse und Form des abgesprengten Stückes ist bohnen- bis mandelgross. Man kann diese Verletzung auch experimentell an der Leiche erzeugen (Kragelund). Das abgesprengte Stück kann sofort völlig gelöst, als beweglicher Körper (Corpus mobile; Gelenkmaus) im Kniegelenk sich befinden; wahrscheinlich gibt es auch Fälle, in denen das Stückchen noch mit Fasern oder Lamellen am Knochen lose haftet und erst allmählich durch wiederkehrenden Druck und Verschiebung (bei aktiver Bewegung) völlig gelöst wird, um dann auch als "Gelenkmaus" sich zu verhalten.

Für die rein traumatische Natur in weitgehendem Sinne ist vor allen Barth (8) eingetreten. Er meisselte Tieren nach Eröffnung des Knie- oder Ellenbogengelenkes Stücke der Grelenkflächen ab und liess diese im Gelenke. Bei diesen künstlichen Versuchen an Tieren wie auch bei allen Leichenexperimenten war stets eine enorme Gewalt nötig, wie sie ein gesundes Gelenk kaum je erleidet. Barth kommt in seiner Arbeit zu der Überzeugung, dass neben der Entstehung in einem durch Arthritis deformans veränderten Gelenke nur die traumatische Absprengung eines Teiles der Gelenkoberfläche 
in Betracht komme. "Andere Entstehungsweisen, wie sie in zahlreichen Hypothesen einer früheren Zeit behauptet worden sind und zum Teile auch heute noch gelten, sind pathologisch-anatomisch nicht nachweisbar, während nach meiner Überzeugung sämtliche Gelenkkörper anatomisch in den beiden vorhergenannten Gruppen unterzubringen sind." Barth glaubt, durch seine Untersuchungen die Lehre von der Entstehung der Gelenkmäuse im wesentlichen zum Abschlusse gebracht zu haben. Der Autor sagt, es läge auch allemal da ein Trauma vor, wo anamnestisch nichts zu ermitteln sei. Es ist aber an sich unwahrscheinlich, dass besonders bei unserer Unfallgesetzgebung jemand ein solches Trauma und seine Folgen ganz vergessen sollte, da doch schon die Quetschung der bedeckenden Weichteile schmerzhaft und der Erguss von Exsudat oder Blut in die Gelenkhöhle dem Betreffenden meist auffällig ist. Während viele Autoren für eine Reihe von Fällen, da ein Trauma nicht offenkundig ist, und da das Gelenk auch durch Arthritis deformans nicht verändert war, Nekrose und Elimination des Knorpels oder Knochens annehmen, behauptet $\mathrm{Barth}$, dass in all diesen Fällen ein Trauma vorliegen müsse. Er sagt: „Ein Körper, der zum grössten Teile aus normalem lebendem Gelenkknorpel besteht, kann nicht durch einen Nekrosierungs- oder Eliminationsprozess entstanden sein, man müsste denn unsere ganzen Begriffe von Leben und Tod der Körpergewebe beiseite schieben. Die Schwierigkeiten liegen in der Beurteilung der sekundären Veränderungen, welche ausgesprengte Gelenkteile mit der Zeit erleiden. Darauf geht in erster Linie die Untersuchung." Der Autor kann auf Grund mikroskopischer und klinischer Beobachtungen keinen Anhaltspunkt finden, etwas anderes anzunehmen, als das, was ein Trauma beim Tierversuche zustande bringen konnte. Nun muss aber Barth selbst sagen: „Bisher ist es nicht gelungen, solche künstlichen Gelenkkörper länger als ca. 6 Monate im Gelenke zu erhalten." Während er in einem Falle von durch Trauma erzeugtem Gelenkkörper die Übereinstimmung der sekundären Veränderungen in produktiver und degenerativer Beziehung mit denen, die bei künstlich abgesprengten Körpern beobachtet wurden. hervorhebt, muss er doch zugeben, dass die auf eine Resorption des Körpers abzielenden Vorgänge im Vergleiche zu den korrespondierenden Stadien im Tierversuche hier sehr in den Hintergrund treten. Bei den durch ein Trauma erzeugten Gelenkkörpern überwiegt vielmehr die Tendenz der Gewebsneubildung, den Körper zu einem bleibenden und selbständigen Gebilde zu gestalten, und der Gedanke liegt nahe, dass nach Fertigstellung der soliden Knorpeldecke an der Knochenbruchfläche der Körper sich unter dem Einflusse der Gelenkbewegungen allmählich gelockert und gelöst habe und $\mathrm{zu}$ einem freien Körper geworden sei. Übereinstimmend sind im Tierversuche und in den Fällen beim Menschen:

1. Der Gelenkknorpel bleibt am Leben, der knöcherne Anteil stirbt ab.

2. Die Bruchfläche des ausgesprengten Stückes erleidet ähnliche Veränderungen wie im Tierversuche; sie wird entweder durch ein osteoides oder noch häufiger durch ein Knorpelgewebe abgeschlossen und von einem Bindegewebsmantel bedeckt. Von der Gelenkwand wächst ein junges Gewebe in 
die Bruchfläche hinein und organisiert sich zu Knorpel- und Knochengewebe d. h. in den Fällen, da eine Verwachsung mit der Gelenkwand da ist, in den anderen Fällen ist die Herkunft dieses Gewebes fraglich.

Die Frage der sekundären Veränderungen der abgelösten und frei in der Gelenkhöhle liegenden Körper in produktiver oder degenerativer Beziehung ist eben noch eine offene Frage. Virchow sagt, dass man manche Petrifikation als etwas Sekundäres betrachten dürfe. Ob ein wirkliches Wachstum stattfinde, sei schwer zu sagen. Wohl komme Vergrösserung durch Umlagerung von Exsudat oder von Blutgerinnseln vor, Konglomeratbildung könne den Eindruck des Gewachsenen machen. Vielleicht aber werde eine Vita minima geführt, vielleicht bestände sogar ein regerer Lebenszustand, indem aus der Synovialflüssigkeit nicht nur Ernährung, sondern auch Wachstum stattfinde. Aber nirgends ist davon die Rede, dass degenerative Prozesse vorwiegen, dass wie im Tierversuche nach ca. sechs Monaten alles verschwunden wäre.

Man kann sich also nicht wundern, wenn die Mehrzahl der Forscher für die Fälle, da ein reines Trauma nicht offenkundig ist, ebenso wie bei den durch Arthritis deformans bedingten Gelenkveränderungen pathologische Prozesse annimmt. König ist es, der in solchen Fällen von Osteochondritis dissecans spricht. Sein Vorgänger in dieser Ansicht ist Broca. Dieser nimmt für sonst nicht zu erklärende Fälle eine bald oberflächliche, bald tiefe Nekrose der Knorpel an und lässt die freien Gelenkkörper als Produkte dieses Eliminationsprozesses erscheinen. Eine Ablösung gibt auch Virchow zu, aber es sind nach ihm das nur Knorpelblätter, flache Stücke, nicht jene dicken Stücke, wie man sie bei der Betrachtung der freien Gelenkkörper im Auge hat. Während die Exfoliation vornehmlich an der Artikulationsfläche stattfindet, geht die Bildung der Körper von den Stellen aus, wo auch die deformierende Entzündung am stärksten ist, am Umfange des Gelenkknorpels, am Periost und in der Synovialis. In jedem Falle aber sind sie, wie Virchow sagt, das Erzeugnis irritativer Prozesse. Der eigentliche Urheber von der Lehre der Osteochondritis dissecans ist aber König. Er versteht hierunter einen zirkumskripten entzündlichen Prozess, der besonders bei jugendlichen Individuen vorkommt, und dessen Tätigkeit darin besteht, Stücke der Gelenkfläche allmählich ausser Kontakt mit ihrer Umgebung zu bringen, wobei eine Verdrehung oder ein leichtes Trauma die vollständige Loslösung bewirkt. König (9) unterscheidet zwei Stadien des Prozesses und gibt in pathologischanatomischer Beziehung an, dass im ersten Stadium die Gelenkoberfläche nicht wesentlich verändert ist; das in Lösung begriffene Stück liegt noch fest der Oberfläche an, nur läuft ringsum ein Knorpelspalt. Mit fortschreitender Lösung wird es beweglicher, zur Seite verschoben oder in die Höhe gehoben. Unter ihm sieht man die Oberfläche des Defektes durch Granulationen erweicht und den Knorpelrand wie zernagt; bald aber tritt eine Glättung auf, welche gleichen Schritt mit der nach dem Zentrum fortschreitenden Lösung hält, so dass schliesslich nur noch ein breiter glatter Stiel Körper und Defekt verbindet. Dieser wird länger und dünner und reisst schliesslich durch. Der 
Prozess ist damit in das zweite Stadium getreten. Die Bruchfläche ist Knorpel oder Knochen. Letzterer wird stets bald nekrotisch, ersterer meist nicht. Die Synovialis in der Nähe des erkrankten Gebietes ist verdickt und sammetartig gerötet, manchmal mit stärkeren flottierenden roten Zotten besetzt. Des weiteren präzisiert der Autor (10) seine Ansichten folgendermassen:

1. „Durch eine Verletzung herbeigeführte Ablösung von Teilen der Gelenkoberfäche, welche als freie Gelenkkörper in der Folge auftreten, ist verhältnismässig selten und bei gesunden Gelenken nur als Folge schwerer Gewalteinwirkung denkbar.

2. Fs können infolge solcher schweren Gewalteinwirkung Stücke der Gelenkoberfläche durch die Fixationsbänder ausgerissen oder ganze Abschnitte einer Gelenkoberfläche, wie der Radiuskopf, das Caput femoris durch Hebelwirkung wie durch zertrümmernde Gewalt oder auch durch dieselben Gewalteinwirkungen ein seitliches Stück abgebrochen werden. Dagegen ist es schlechterdings undenkbar, dass flache Stücke aus der Oberfläche eines artikulierenden Knoçhenendes, wie wir sie aus dem freien Ellenbogengelenk, aus dem Knie beschrieben haben, durch einen Verletzungsakt sofort ohne anderweitige schwere Verletzung des Gelenkes abgesprengt werden.

3. Es ist sehr wohl denkbar, dass Stücke der Gelenkfläche bei einer Verletzung kontundiert werden, so dass Nekrose derselben und eine nachträgliche dissezierende Entzündung, die zu ihrer Ablösung führt, eintritt.

4. Es gibt eine spontane Osteochondritis dissecans, welche ohne sonstige nennenswerte Schädigung des Gelenkes beliebige Stücke der Gelenkoberfläche zur Lösung bringt. Ein grosser Teil der bis jetzt als traumatisch aufgefassten Fälle von freien Gelenkkörpern muss ais auf diesem Wege entstanden gedacht werden.

5. Die Ätiologie des gedachten pathologisch-anatomischen Prozesses ist vorläufig noch unbekannt.“

Weiter sagt derselbe Autor: „Wir sind uns wohl bewusst, dass wir über das Wesen des Prozesses nichts sagen, wenn wir annehmen, dass die abgestorbenen Körper durch eine Osteochondritis frei geworden sind. Die Vorstadien, bezw. die Entwickelung der Krankheit sind nicht bekannt; wir kennen nur den Effekt, der darin besteht, dass entweder grössere oder kleinere Knorpelstücke aus den sonst ganz intakten Gelenkenden losgelöst werden, so dass sie zu freien Gelenkkörpern werden, oder dass grössere Teile des Knochens, also ganze Schenkelköpfe abgetrennt werden. Erst wenn durch einen glücklichen Zufall die verschiedenen, der Lösung vorhergehenden Veränderungen der Knochen zu unserer Kenntnis gekommen sein werden, können wir uns ein Urteil über die bis dahin so rätselhafte Osteochondritis dissecans machen."

In einem Falle sagt König: „Im vorderen Ende des Defektes, in den der beschriebene Körper genau hineinpasst, liegt noch ein kleines Stück ohve Knorpelüberzug, welches das Aussehen eines nekrotischen Knochens hat. (Sequester.)" 
Die Mehrzahl der Forscher gibt im grossen und ganzen König recht, wenigstens werden sie alle nicht durch die zwei ätiologischen Nomente reines Trauma, Arthritis deformans - befriedigt. Virchow sagt in seiner Geschwulstlehre: „Die deformierende chronische Entzündung kommt auch ohne alle Gicht, ohne. Störungen der sensitiven Nerven, bei jungen Personen und ganz partiell auf kleine Stellen des Gelenkes beschränkt vor. Diese Form ist es, welche der Bildung der solitären Gelenkkörper zugrunde liegt."

In den Körpern, die aus Knorpelknochen bestehen, hat man die Rinnen zwischen den Balken öfters mit Markfett angefüllt getroffen. Dies beweist, dass früher eine ausgiebige Verbindung des Körpers mit anderen Teilen des Gelenkes stattgefunden haben muss, da eine solche Markbildung stets eine starke Vaskularisierung voraussetzt. Für solche Fälle gibt Virchow die Möglichkeit zu, dass wirklich Teile der Knochengelenkenden sich ablösen und Knorpel und Knochen in die Gelenkhöhle gelangen.

Hildebrand (11) verhält sich ebenfalls ablehnend gegenüber der Annahme eines reinen Traumas. Die Resultate seiner Versuche fasst dieser Forscher folgendermassen zusammen: „Das Resultat dieser Versuche war also, dass sechs gestielte Gelenkkörper resorbiert waren, ebenso fünf freie, während vier freie mit der Kapsel verwachsen und erhalten geblieben waren. Nicht ein einziger war also als freier Gelenkkörper erhalten."

Weiter sagt Gies (12): "Meine Experimente beweisen mir, dass diese Knorpelstückchen nach kürzerem oder längerem Verweilen im Gelenke regressiven Metamorphosen unterliegen. Die beiden Patellen im fünften Versuche sind sogar gänzlich resorbiert, mithin scheint mir der Beweis erbracht, dass die freien Gelenkkörper - sensu strictiori - sich nicht durch traumatische Ablösung der Gelenkflächen entwickeln." Seine Theorie lautet: „Die freien Gelenkkörper sind als eigentliche Neubildung, Enchondrome aufzufassen." Er gesteht aber zu, dass durch intrakapsuläre Frakturen abgesprengte Knochenstücke den Symptomenkomplex einer Gelenkmaus erzeugen können. Der Autor führt weiter aus: „So untersuchte H. v. Meckel ein abgebrochenes Stück des Processus coronoideus ulnae, das als Fremdkörper aus dem Ellenbogengelenk exzidiert worden war. Der Fall von Recklinghausen und der von Hase lassen ebensowenig über die Fntstehungsart der fraglichen Gelenkkörper durch ein Trauma - intrakapsuläre Fraktur - irgendwelche Zweifel obwalten. Wir sind eben über den Entstehungsmodus in vorher gesunden Gelenken unklar."

Real (13) sagt am Schlusse des Berichtes über neun Fälle: „Es ist hervorzuheben, dass für keinen derselben die rein traumatische Entstehung sicher erwiesen ist."

Der dänische Forscher Kragelund (14) führt 12 von ihm selbst genau beobachtete Fälle an, die für einen osteochondritischen Prozess sprechen. Er sagt, spontan könne eine solche Osteochondritis dissecans Stuicke von Gelenkflächen ablösen, ja ganze Gelenkköpfe könnten abgelöst werden. „Das Trauma bewirkt eine Ernährungsstörung in derjenigen Ausdehnung, in welcher der Schlag bei Leichenversuchen eine Fraktur verursacht. Durch eine sich an- 
schliessende Ostitis wird der Zusammenhang der abgesprengten Partieen mit der Umgebung vermindert, und später löst sich der Sequester und wird zur Gelenkmaus."

Klein (15) spricht in einem auf andere Weise nicht erklärbaren Falle von einer spontanen Demarkation.

Volkmann (16) sagt: „Einzelne zirkumskripte Partieen des Gelenkknorpels hören auf, ernährt zu werden und stossen sich ab."

Die französischen Forscher Poulet und Vaillard (17) stimmen fast ganz den Ansichten $\mathrm{K}$ önigs bei, indem sie sagen:

"On sait, en effet, qu'il existe certaines formes d'arthrite sèche, non seulement monoarticulaires mais encore très limitées à un point circonscrit d'une seule jointure; celles-ci peuvent survenir chez des sujets jeunes, et évoluer sans troubles fonctionnels bien accusés. Telle est sans doute la forme, qui donne lieu aux corps étrangers solitaires."

Und weiter pag. 298:

„Enfin il existe une maladie indeterminée, à marche insidieuse, qui provoque l'élimination de fragments " plus ou moins volumnieux des tets osseuses."

Und schliesslich pag. 291 :

"Ceux-ci seraient alors produits par une affection osseuse, sourde, silencieuse, localisée en un point des epiphyses et d'essence inconnue encore; ils se recontrent chez des individues relativement jeunes et résultent de la séquestration de fragments ostéo-cartilagineux; etc."

James Paquet nennt den Vorgang quiet necrosis.

Der ganze Streit scheint solange nach der einen oder anderen Seite hin nicht entschieden werden zu können, als wir nicht mikroskopisch die Histologie der einen durch ein reines Trauma entstehenden Art von den Gelenkkörpern trennen können, welche eine spontane Osteochondritis dissecans geschaffen hat.

In der Mehrzahl der Fälle handelt es sich aber um das Auftreten von Gelenkkörpern in erkrankten Gelenken, deren Affektion wir genau kennen. Ein Trauma ist dann nur die Gelegenheitsursache, um die geschaffene Exkreszenz frei zu machen. Von den hierher gehörigen pathologischen Prozessen kommt in erster Linie die Arthritis deformans in Betracht. Hierunter versteht man nach Schmaus die wesentlich mit starken Wucherungs- und Zerfallserscheinungen an den Gelenkenden sowie mit Wucherungen der Synovialis einhergehenden Formen der chronischen Gelenkentzündung. Auch die chronische seröse oder serofibrinöse Arthritis kann Veranlassung zur Bildung freier Gelenkkörper sein. Dabei wird ein dünnflüssiges oder dickeres, mehr kolloides Sekret in die Gelenkhöhle abgeschieden. In der Synovialis stellen sich Wucherung der Zotten, pannöse Wucherung über dem Knorpel und fibröse Verdickung ein. Auch Ziegler (18) steht auf diesem Standpunkte, indem er sagt: „Die wichtigsten freien Gelenkkörper sind diejenigen, welche durch Loslösung hypertrophischer Wucherungen, wie sie bei Arthritis deformans vorkommen, entstehen und am häufigsten aus Knorpel aufgebaut 
sind, der durch Wucherung von in den Zotten und in der Gelenkkapsel befindlichen Knorpelherden entstanden ist."

Ein Trauma kann aber auch die Veranlassung zum Auftreten der Arthritis deformans geben, deren Wucherungen dann im Verlaufe der Krankheit durch ein weiteres Trauma abgesprengt werden können. $\mathrm{Zu}$ diesen seltenen Fällen, Erzeugung der Arthritis deformans durch ein Trauma gehört der von Huber (19) beschriebene Fall. Ein 59jähriger Mann hatte vor 30 Jahren einen Unfall. Er war durch herabfallende Lehmmassen verschüttet; das linke Bein, besonders das linke Kniegelenk war affiziert. Von dem Unfalle ab datiert sein immer grössere Beschwerden verursachendes Leiden. Das Trauma war die Veranlassung zu einer chronischen deformierenden Gelenkentzündung (Arthritis deformans), in deren Verlaufe ossifizierende Knorpelwucherungen und Ablösungen von solchen zur Bildung von Gelenkmäusen führten. Hoffa entfernte aus beiden Kniegelenken freie Gelenkkörper und konstatierte daneben Unebenbeiten und Protuberanzen an den Gelenkenden (Arthritis deformans).

$\mathrm{Zu}$ arthritischen Wucherungen und damit zur Bildung von Gelenkmäusen gibt besonders die Arthritis der Tabiker Veranlassung. Bei diesen Kranken kommt ausserdem eine besondere Brüchigkeit der Knochen in Betracht.

Auch die Rachitis prädisponiert zur Bildung von freien Gelenkkörpern. Die lipomatöse Entstehungsweise ist ebenfalls sehr klar. v. Rindfleisch (20) sagt hierüber: „Die Synovialis neigt zur Bildung papillöser Exkreszenzen. Die schon in normalen Gelenken vorkommenden Gelenkzotten können einen grösseren Teil des Synovialsackes namentlich dann ausfüllen, wenn sich in den Papillen Fettgewebsträubchen ausbilden, die das Volumen derselben beträchtlich vergrössern (Lipoma arborescens Mülleri).“

Volkmann beobachtete eine Bildung von knorpeligen Platten in der Synovialis selbst, welche dann gleichfalls zur Ablösung kamen, und Virchow erwähnt in seiner Geschwulstlehre den Übergang gewucherter Papillen und Zotten der Synovialis in sklerotisches Bindegewebe, ja sogar Übergänge in Knorpel, alles Bildungen, die dann gelegentlich durch ein Trauma zu freien Gelenkkörpern werden können.

Schon normalerweise finden sich in den Zottenenden. Knorpelkerne, wie es Koelliker und Luschka ausgeführt haben.

Dass auch vom Perioste Knorpelwucherungen ausgehen können, hat zuerst Laennec beobachtet. Zuerst ausserhalb des Gelenkes liegend, stülpen sie sich mit der Zeit in dieses ein. Der Autor sagt hierüber, „qu'ils se développent à l'exterieur des articulations et qu'ils enfoncent peu-à-peu dans leur interieur enveloppés par un prolongement des membranes synoviales, celui-ci se rompe dans quelque mouvement ou par une sorte d'usure, et le corps cartilagineux se trouve libre dans la cavité articulaire."

Für einige seltene Fälle wird die Bildung aus Fibrinniederschlägen nach Blutungen oder fibrösen Entzündungen angegeben. Coagula können von jungem Bindegewebe durchwachsen und nach Art thrombotischer Massen organisiert werden. Diese Ansicht ist schon vor langer Zeit von John $\mathrm{Hunter}$ 
vertreten worden, wonach die freien Gelenkkörper amorphe Konkretionen aus geronnenen, extravasierten oder exsudierten Massen seien. In ihrer Allgemeinheit lässt sich aber nach obigen Ausführungen diese Ansicht nicht halten, und Virchow beschränkt sie, indem er sagt, es liesse sich nicht in Abrede stellen, dass für manche Gelenkmäuse die Ansicht Hunters zutreffen möge.

F ischer beobachtete, wie es durch Fall auf das Kniegelenk zu Hämarthros und später durch Gerinnung zur Bildung von Gelenkkörpern kam. Eine spontane Heilung wurde beobachtet, weshalb der Autor solche Bildungen für sehr vergänglich ansieht.

Alle nicht aus Knochen bestehenden Körper können petrifizieren, zu Arthrolithen werden.

Freie Körper finden sich aber nicht nur in den Gelenkhöhlen, man findet sie auch zuweilen in den Bursen über Exostosen. Da solche Bursen nach der unten weiter auszuführenden Hypothese von Rindfleisch Ausstülpungen der Gelenkkapsel sind, so stehen diese freien Körper auch mit den freien Gelenkkörpern in Verbindung. Diese Art freier Körper soll daher hier noch näher beschrieben werden, und an der Hand eines hier in Würzburg beobachteten Falles von Exostosis bursata soll die Berechtigung der Anschauung von $\mathrm{Rindfleisch} \mathrm{nachgewiesen} \mathrm{werden.}$

In neuester Zeit hat Riethus (21) über einen Fall von Exostosis bursata mit freien Knorpelkörpern berichtet. Den Begriff Exostosis bursata hat Volkmann 1869 für jene seltenen Bildungen vorgeschlagen, bei denen es sich darum handelt, dass ein mit Knorpel überzogener Teil einer Exostose in einen Hohlraum hineinragt, der von einer derben bindegewebigen Kapsel umgrenzt und mit einer synoviaähnlichen Flüssigkeit angefüllt ist. Wie Riethus angibt, sind nur 17 derartige Fälle bekannt. In der meist klaren, synoviaartigen Flüssigkeit, die nach Trauma hämorrhagisch, in einem anderen Falle eiterig infiziert gefunden wurde, sind in seltenen Fällen in der Flüssigkeit suspendierte freie Körper in grosser Anzahl gefunden. Die erste Beobachtung dieser Art stammt von Billroth und wurde 1864 ausführlich von $\mathrm{Rindfleisch}$ beschrieben. In dem von $\mathrm{Ri}$ ethus mitgeteilten Falle handelt es sich um einen 16 jährigen Bierverleger, der sich beim Heben eines Fasses gegen das rechte Knie stiess. Am anderen Morgen fiel an der Innenseite der rechten Kniegelenkgegend eine bisher noch nicht beobachtete hühnereigrosse Anschwellung auf, die schmerzlos und nicht hinderlich bei Bewegungen war. Die Anschwellung nahm in der Folgezeit zu, so dass der Junge die Leipziger Klinik aufsuchte. Hier ergab die Röntgenuntersuchung, dass es sich um einen Fall von multiplen Exostosen handelte. An der Basis der am Condylus internus tibiae bestehenden Geschwulst erkennt man deutlich eine der Tibia aufsitzende Exostose, in deren Umgebung eine Anzahl kleinerer, nicht besonders scharfer Schatten von unbestimmter Form zu sehen sind; nur ein grösserer scheint dicht an der Spitze der Exostose zu liegen. Durch Operation wurde die ganze Geschwulst bis zur Basis stumpf isoliert, die 
Exostose an der Basis mit dem Meissel abgeschlageu und die Geschwulst in toto entfernt. Wie die Untersuchung des Präparates ergab, ragen in das Innere der Cyste hinein erstens die Exostose und zweitens gegenüber der Spitze der Exostose ein analoges Gebilde, das ebenso wie jene Exostose in der Mitte eine spongiöse Knochenmasse erkennen lässt, die am Rande von einer mehr kompakten Schicht begrenzt wird. Dieses Gebilde wurde als das periphere Bruchstück der Exostose erkannt. Demnach hat sich in diesem Falle die Bursa nicht über dem peripheren Teile einer Exostose entwickelt, sondern zwischen den beiden Fragmenten einer abgebrochenen Exostose. Riethus sieht daher die Bursa für eine sekundäre Bildung an: Durch das Trauma ist eine Fraktur der bereits bestehenden kartilaginären Exostose an dem Condylus internus tibiae entstanden. Infolge der fortgesetzten mechanischen Insulte, wobei das periphere Fragment immer wieder verschoben wurde, kam es nicht zur Konsolidierung der Fragmente, sondern zur Bildung einer Pseudarthrose. Zwischen beiden Fragmenten trat keine solide bindegewebige Vereinigung ein, sondern durch Wucherung des an den Bruchflächen frei liegenden Knorpelgewebes entstanden Ekchondrosen, die mechanisch von ihrer Unterlage losgerissen sich zwischen die Fragmente und das die Exostose einhüllende Gewebe drängten. So entstand allmählich um die beiden Fragmente eine Bindegewebskapsel, die in einer serösen Flüssigkeit eine grössere Anzahl freier Knorpelkörper enthielt. In der isolierten Cyste fanden sich die allermanigfaltigsten freien Körper. Frei lagen darin vier grössere von dem Umfange einer welschen Nuss, daneben eine Menge bedeutend kleinerer Körper bis zur Feinheit eines Stecknadelkopfes. Sie schienen zum Teil der Wandung fester anzuhaften und lagen förmlich eingedrückt in die Zotten an der Innenfläche der Wand, zum Teil füllten sie, in mehreren Lagen übereinanderliegend, den Raum zwischen den grösseren Körpern aus. Ihre Zahl betrug an 200.

Wie oben erwähnt, stellt Riethus für seinen Fall die Entstehung der Bursa als einen sekundären Prozess hin und hält seine Beobachtung für etwas Neues, wie es noch nicht gesehen wurde. Er stellt für seinen Fall seine eigene, oben angegebene Erklärung auf und stimmt damit nicht überein mit der Annahme, welche Rindfleisch bei der Beschreibung des Billroth'schen Falles gemacht hat. Rindfleisch fand eine volle Übereinstimmung der Wand der Bursa, in der 38 freie Knorpelkörper gefunden wurden, mit den Elementen einer echten Synovialmembran und stellte die Bildung der freien Körper analog den bei Arthritis deformans am Gelenkknorpel beobachteten Vorgängen. Die Hypothese, die Rindfleisch für die Genese der Exostosis bursata aufgestellt hat, lautet:

Die Exostosis bursata stellt nicht, wie die gewöhnlichen kartilaginären Exostosen eine Ekchondrose des Intermediärknorpels dar, sondern sie ist eine Ekchondrose des Gelenkknorpels, entstanden intraartikulär an der Insertionsstelle der Gelenkkapsel. Im weiteren Verlaufe stülpt die Exostose die Gelenkkapsel vor sich her, genau so, wie beim Descensus testiculi der Testis das Peritoneum vor sich her stülpt. Der Recessus der Gelenkkapsel über 
der Exostose schnürt sich auch vollständig ab, und, indem er sich rings an der Basis der Exostose ansetzt, bildet sich auf diese Weise die Bursa, eine Ausstülpung der echten Gelenkkapsel.

Für die Berechtigung dieser Anschauung von Rindfleisch spricht folgender von Herrn Professor Riedinger operierte Fall. Herr Privatdozent Riedinger hat über denselben in der physikalisch-medizinischen Gesellschaft zu Würzburg in der Sitzung vom 16. Juli 1903 berichtet.

Während in obigem Falle von Riethus das Trauma an der Exostose als an einer schon bestehenden Bildung angreift und die Bursa für etwas Sekundäres aufgefasst wird, entsteht in unserem Falle erst durch das Trauma die Exostose, genauer Kallus, und das Trauma hat ein Stück der Gelenkkapsel, die spätere Bursa ausgerissen.

Die 47 jährige Bauersfrau H. D. von W., die früher stets gesund war und innerlich gesund ist, erlitt anfangs September 1901 einen Unfall. Die Frau führte mit der rechten Hand ein Kalb an einer Kette. Das Tier machte eine plötzliche Bewegung und zog heftig an der Kette. Die Frau verspürte dabei einen Riss am Vorderarme und einen starken Schmerz. Alsbald trat am volaren oberen Teile des rechten Vorderarmes eine starke Schwellung auf, deren Bedeckung sich nach einigen Tagen gelbgrünlich verfärbte. Auf ärztliche Verordnung wurden Umschläge und Einreibungen gemacht, doch wurden die Schmerzen immer ärger, und die Geschwulst wurde allmählich härter. Im November 1901 vermutete der Arzt Flüssigkeit und machte einen Einstich. Anfangs waren die Bewegungen im Gelenke stark behindert, doch allmählich lernte die Patientin Bewegungen wieder langsam ausführen. Schmerzen waren auch nachts vorhanden, doch immer nur an der Verletzungsstelle. $\mathrm{Ob}$ das Ellenbogengelenk an der Schwellung beteiligt war, weiss Patientin nicht.

Bei der klinischen Untersuchung im November 1902 wurde eine Geschwulst zweifingerbreit unterhalb der Gelenkbeuge am rechten Vorderarme konstatiert. Die Geschwulst war kugelig, ca. $1-2 \mathrm{~cm}$ breit und ebenso hoch. Sie fühlte sich hart an. Die äussere Haut war unverändert, verschieblich, ohne Entzündungserscheinungen. Wie das Röntgenbild erläutert, sass die Geschwulst der Tuberositas radii unmittelbar auf. Auf der Geschwulst und seitlich sieht man mehrere kleine, undeutliche Schatten. Bei Bewegungen und bei Druck klagte die Patientin über Schmerzen.

Durch einen Längsschnitt parallel dem Radius wurde die Geschwulst freigelegt. Dieselbe lag in einer Höhle eingebettet, aus der Gelenkflüssigkeit deutlich abfloss. Durch Emporhebung der Weichteile oder durch Einführung einer Sonde konnte die Kommunikation mit dem Ellenbogengelenk durch einen etwa $1 \mathrm{~cm}$ breiten, taschenförmigen Gang nachgewiesen werden. Das Ellenbogengelenk lag somit frei. Die Bicepssehne war retrahiert. Die Oberfläche der Geschwulst hatte das Aussehen des Gelenkknorpels und die Wandung der Höhle das der Gelenkkapsel. Ausserdem fanden sich sechs freie Körper, die als Gelenkmäuse imponierten, stecknadelkopf- bis bohnengross. Sie sind auch auf dem Röntgenbilde zum Teil zu erkennen. Der dem oberen 
Gelenkende des Radius knöchern aufsitzende Tumor wurde abgemeisselt, und die freien Gelenkkörper wurden entfernt. Es erfolgte dann eine glatte Heilung mit Funktionsfähigkeit des Armes.

Die von Herrn Professor Dr. Borst geleitete Untersuchung zweier freier Körper aus der Geschwulst ergab folgende Resultate:

Der grösste, annähernd kugelige freie Körper war an seiner Oberfläche glatt, leicht buckelig und hatte ungefähr die Konsistenz des Knorpels. Auf einem Durchschnitte erschien der Körper wie von einer peripheren bindegewebigen Membran überzogen, während im Zentrum eine homogene, knorpelige, rundliche Stelle gefunden wurde. Das von der Exostose abgemeisselte Stück zeigte ebenso eine periphere wie periostale Begrenzung, sonst bestand wenigstens für die makroskopische Untersuchung der ganze Körper aus spongiösem Knochen. Die beiden Gebilde wurden nun entkalkt und nach Celloidineinbettung in verschiedentlicher Weise gefärbt. Die mikroskopische Untersuchung des abgemeisselten Stückes ergab schon bei der oberflächlichsten Betrachtung, dass man es mit einer gewöhnlichen Exostose jedenfalls nicht zu tun hatte. Zwar bestanden die zentralen Teile dieses Gebildes aus typischem lamellärem Knochen, der zu einer sehr weitmaschigen Spongiosa angeordnet war. In den sehr weiten Markräumen dieser Spongiosa traf man ausser auf die Gefässe auf ein fettiges oder gallertiges Markgewebe. Die Knochenbälkchen selbst zeigten typische Knochenkörperchen, die nach Gestalt und Anordnung, sowie auch nach ihrer Zahl durchaus die Verhältnisse des normalen ruhenden Knochens darboten. Hie und da sah man in einem breiteren Knochenbälkchen auch ein $\mathrm{H}$ avers'sches Kanälchen mit sehöner konzentrischer Schichtung der Knochenlamellen um dasselbe. So waren aber nur die allerinnersten Teile der "Exostose" gebaut. Ging man weiter nach aussen, so trat hier eine ausgebreitete Knochenneubildung auf. Diese ging einmal von den Markräumen des Spongiosamaschenwerkes aus, dann aber von einer sehr breiten, den ganzen Körper umhüllenden, wuchernden, periostalen Bindegewebsschicht Von den Markräumen aus erfolgte eine Anlagerung von Osteoblasten an die Knochenbälkchen, von Osteoblasten, welche oft in einer doppelten Reihe seitens des wuchernden, hier sehr zell- und gefässreichen Markgewebès abgesetzt wurden. Durch die Tätigkeit der Osteoblasten wurden ziemlich breite Schichten jungen Knochengewebes auf die bereits verknöcherten oder sich noch im osteoiden Zustande befindlichen Knochenbälkchen abgelagert. Je näher man gegen die Peripherie des hier zu beschreibenden Gebildes vorrückte, desto mehr wurde die Spongiosa nicht von knöchernen, sondern von osteoiden Bälkchen gebildet, in denen man da und dort die ersten Ansätze der Verkalkung beobachtete. Ganz an der Peripherie traf man Bälkchen von chondroidem.Charakter, in welchen ebenfalls die Ablagerung der Kalksalze im Beginne war. Diese Knorpelbälkchen gingen ebenso wie solche von mehr osteoidem Charakter aus einer Metaplasie des vorerwähnten wuchernden periostalen Bindegewebes hervor. Dieses war ein an grossen langgestreckten und rundlichen Zellen sehr reiches fibrilläres Gewebe, in welchem die fibrilläre Substanz da und dort $z u$ einer homogenen Masse 
zusammensinterte, wobei dann die eingeschlossenen Zellen entweder in kleine zackige Höhlen zu liegen kamen (osteoide Substanz) oder in grosse rundliche, durch beginnende Verkalkung der Wand noch deutlicher hervorgehobene Höhlen (chondroide Substanz). Unter fortschreitender Verkalkung wurden die so entstandenen Bälkchen schliesslich in echten Knochen übergeführt.

Nach diesem Befunde unterliegt es wohl keinem Zweifel, dass man es mit einem stark proliferierenden osteoblastischen Gewebe zu tun hat, dessen ganze Struktur den sogenannten Kallusbildungen durchaus entspricht.

Der zweite freie, rundliche Körper bestätigte die Vermutung, dass es sich in unserem Falle um eine Kalluswucherung handeln möchte, auf das beste. Auch dieser Körper war von einer peripheren, ziemlich beträchtlichen Bindegewebsmembran überzogen. Diese Membran bestand aus einem an Zellen wechselnd reichen fibrillären Bindegewebe, dessen Fügung hier ausserordentlich dicht und straff, dort locker, feinmaschig erschien. An vielen Stellen ging dieses Bindegewebe kontinuierlich in Knorpelsubstanz oder in osteoide Substanz über. Aus diesen beiden letzteren Substanzen war nämlich der ganze freie Körper aufgebaut. Ganz zentral fand sich der schon bei der makroskopischen Beschreibung erwähnte Knorpelkern: die Knorpelzellen waren sehr gross, die Kapsel ausserordentlich deutlich; stellenweise waren Knorpelgrundsubstanz und die Knorpelkapsel verkalkt. Die Knorpelgrundsubstanz entsprach im grossen und ganzen dem Typus des Faserknorpels, wenn auch da und dort eine durchaus homogene, dem Hyalinknorpel ähnliche Substanz nachgewiesen werden konnte. Die Knorpelzellen waren stellenweise gut erhalten, ihre Kerne deutlich sichtbar, viele Knorpelzellen waren jedoch in beginnendem Zerfalle oder bereits völlig zugrunde gegangen. Dieser Knorpelkern ging unmerklich an der Peripherie ïber in ein osteoides Gewebe, in welchem die Knorpelhöhlen enger wurden und mehr die zackige Gestalt der Knochenkörperchen annahmen. Auch in diesem osteoiden Gewebe war stellenweise Verkalkung eingetreten.

Von grossem Interesse war es nun, zu bemerken, wie sich der ursprünglich offenbar nur aus knorpeliger Substanz aufgebaute solide Körper umzuwandeln begann in einen spongiösen, osteoiden bezw. richtig knöchernen Körper. Dies geschah in der Weise, dass sich in der ganzen Knorpelmasse Bälkchen von osteoidem Charakter herausdifferenzierten, während das zwischen diesen differenzierten osteoiden Bälkchen befindliche Knorpelgewebe unter Verflüssigung und fibrillärer Umwandlung der Grundsubstanz und unter Zugrundegehen bezw. Umwandlung der Knorpelzellen zu grossen sternförmig verästelten Bindegewebszellen sich auflöste und bereits an manchen Stellen zu einem schleimgewebsartigen Markgewebe umgewandelt hatte. Dass auch in diesem freien Körper nicht nur eine Metamorphose des schon vorhandenen Gewebes vor sich ging, sondern auch eine Bildung neuen Gewebes vorhanden war, das ging aus einer Betrachtung der Peripherie unseres Körpers hervor, welche überall einen durch Zellwucherung vermittelten Übergang der den Körper einhüllenden Bindegewebsschicht in junges osteoides Gewebe zeigte. 
Fassen wir den soeben erhobenen anatomischen Befund zusammen und versuchen wir ihn in Einklang zu bringen mit den klinischen Daten, so lässt sich ungefähr folgendes berichten:

Durch das erlittene Trauma kam es zu einer Abreissung des M. biceps von der Tuberositas radii, eine Tatsache, die ja auch später bei der Operation noch durch den Befund der abgerissenen Sehne erhärtet werden konnte. Höchstwahrscheinlich ist bei dieser Abreissung auch die Gelenkkapsel mitverletzt (wabrscheinlich angerissen) worden; im weiteren Verlaufe hat sich nun einerseits an der Stelle der Sehnenabreissung von seiten des mitverletzten Periostes eine Knochenneubildung entwickelt, die auch zur Zeit der Operation noch nicht abgeschlossen war. Dies beweist der Befund von lebhaft wucherndem Kallus im Bereiche der Tuberositas radii. Während also einerseits von der Stelle der Sehnenabreissung eine beträchtliche (traumatische) Exostose entstand, stülpte sich andererseits die verletzte Kapsel des Ellenbogengelenkes über die entstehende Exostose aus, so dass letztere mehr und mehr in den Bereich eines Divertikels der Gelenkkapsel zu liegen kam. Derartige Ektasien nun von Gelenkkapseln nach Verletzungen sind ja häufig genug beobachtet worden. Im weiteren Verlaufe des Prozesses schloss sich der ausgestülpte Teil der Gelenkkapsel mehr und mehr gegen das übrige Gelenk ab. So wurde aus der traumatischen Exostose eine Art von Exostosis bursata. Die Bildung der freien Körper in dem über die Exostose gestülpten Teile der Gelenkkapsel ist, wie die Untersuchung zweifellos ergab, zurückzuführen auf die üppige Kalluswucherung an der Tuberositas radii. Von diesem Kallus luxurians, welcher die bei der Operation gefundene Exostose bildete, hatten sich kleine Teilchen abgelöst und kamen als freie Körper in die Gelenkhöhle zu liegen. Der mikroskopische Befund der freien Körper legte diese Art ihrer Entstehung völlig klar. Es ist interessant, eine Parallele zu ziehen zwischen unserem Falle und dem von Riethus kürzlich berichteten. Während es sich bei Riethus um ein Trauma handelte, welches eine bereits vorhandene typische Exostosis bursata traf, haben wir in unserem Falle sehr deutlich die Entstehungsgeschichte einer solchen Exostosis bursata auf der Basis eines Traumas vor uns. Die Bildung der freien Körper erfolgte allerdings in unserem Falle auf eine ganz andere Weise als in dem Falle von Riethus.

Unser Fall bietet also in chirurgischer wie in pathologisch-anatomischer Beziehung ein grosses Interesse. Er zeigt uns vor allem an einem schönen Beispiele die Entstehung einer traumatischen Exostosis bursata und demonstriert die Richtigkeit der seinerzeit von Rindfleisch ausgesprochenen Behauptung, dass die über solchen Exostosen befindliche Bursa einer Ausstülpung der Gelenkkapsel ihren Ursprung verdankt und zeigt auch zugleich, dass derartige Ausstülpungen durch allmähliche Separation von der übrigen Gelenkkapsel zu mehr selbständigen Bildungen werden können. Nur hat in unserem Falle nicht die Exostose die Gelenkkapsel ausgestülpt, sondern es war die Erweiterung der Gelenkkapsel ebenso wie die Exostose als die Konsequenz einer traumatischen Einwirkung anzusehen, so dass also Exostosis und Bursa zusammen auf die gleiche Ursache zurückzuführen waren. 


\section{Literaturverzeichnis.}

1. Schmaus, Lehrbuch der pathol. Anatomie.

2. Leser, Spezielle Chirurgie.

3. Fischer, Deutsche Zeitschrift für Chirurgie, 1880.

4. Virchow, Die krankhaften Geschwülste, 16. Vorlesung.

5. Fis cher, Deutsche Zeitschrift für Chirurgie, XII, 4. und 5.

6. Schild, Inaug.-Diss., Würzburg 1901.

7. Helferich, Frakturen und Luxationen, 1901.

8. Barth, Langen becks Archiv, Bd. 56.

9. König, Archiv für klinische Chirurgie, Bd. 59, 1899.

10. Derselbe, Deutsche Zeitschrift für Chirurgie, 1887, Bd. 27. Derselbe, Spezielle Chirurgie.

11. Hildebrand, Deutsche Zeitschrift für Chirurgie, Bd. 42 .

12. Gies, Deutsche Zeitschrift für Chirurgie, Bd. 16

13. Real, Deutsche Zeitschrift für Chirurgie, 1893, Bd. 38.

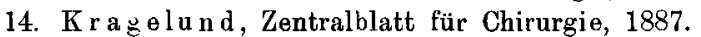

15. Klein, Virchows Archiv, 1864, Bd. 29.

16. Volkmann, Chirurgie von Pitha und Bilìroth und Beiträge zur Chirurgie, 1875.

17. Poulet et Vaillard, Archives de physiologie et patholog., 1885.

18. Ziegler, Spezielle pathol. Anatomie.

19. Huber, Inaug.-Diss., Würzburg 1901.

20. Rindfleisch, Pathol, anat. Gewebelehre.

21. Riethus, Beiträge zur klinischen Chirurgie, Bd. XXXVII, 3. Heft. 
Archiv für Orthopädie, Mechanotherapie und Unfallchirurgie III, 1.

Tafel I.

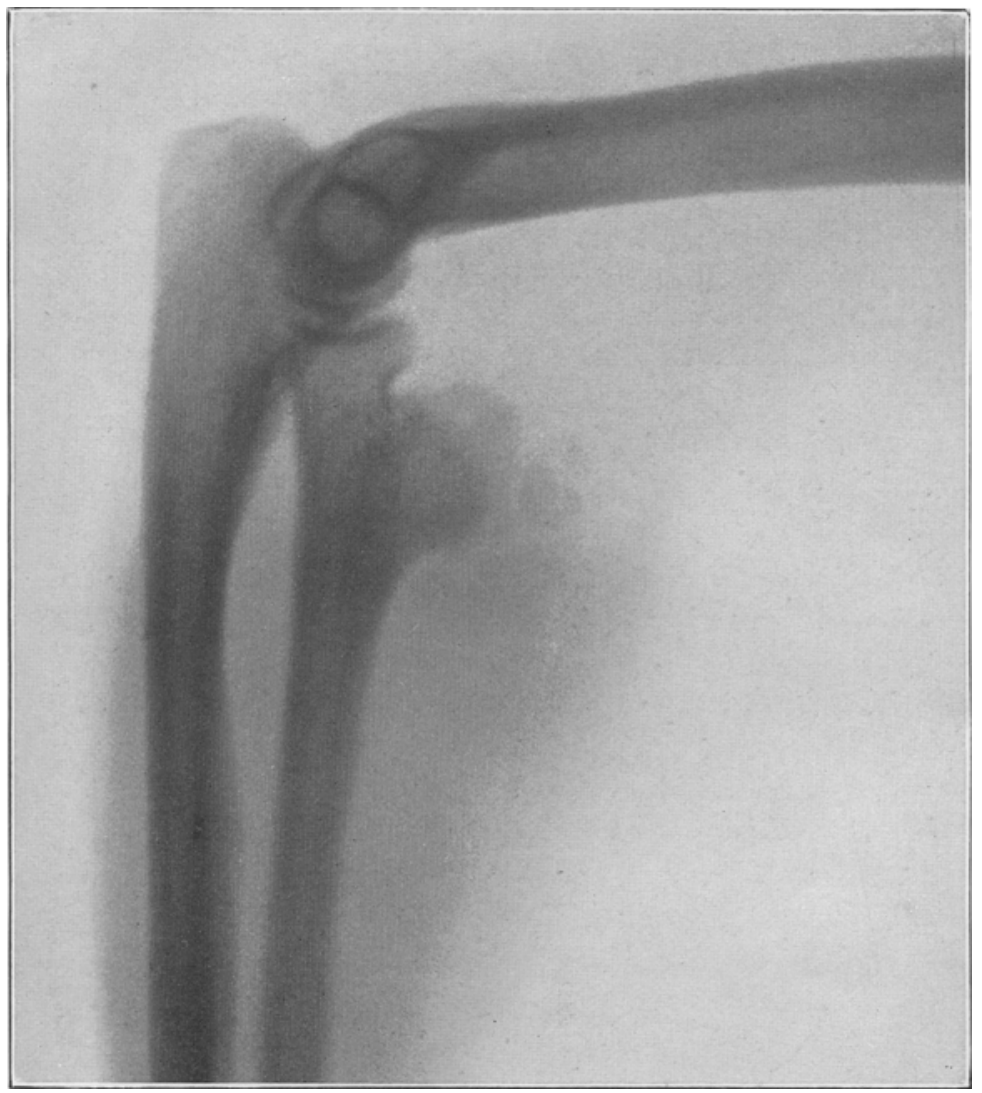

Karl Röper. 